REPLY

\section{Predictive diagnostic tests in periodontal diseases}

\author{
Denis F. Kinane, Panagiota G. Stathopoulou and Panos N. Papapanou
}

We appreciate the opportunity to discuss predictive diagnostics for periodontal diseases further than was possible in our original Primer (Periodontal diseases. Nat. Rev. Dis. Primers 3, $17038(2017))^{1}$ by responding to the recent correspondence by Hernández and colleagues (A quantitative point-of-care test for periodontal and dental peri-implant diseases. Nat. Rev. Dis. Primers 3, 17069 $(2017))^{2}$.

Quantitative diagnostic tests, such as the one the authors propose, which measures the levels of activated matrix metalloproteinase 8 (aMMP8) as a biomarker of active periodontal disease, are welcome new tools and could enhance screening for periodontal disease as well as aid diagnosis and prognostic assessment. However, moreextensive independent verification and a plausible biological argument that supports the role of the selected biomarker in the aetiopathogenesis of periodontal disease are needed before these tests can be recommended for clinical practice ${ }^{3}$. Molecular diagnostics in periodontal disease are to be encouraged, but not prematurely adopted, as occurred with the IL-1 genotype-based diagnostic test ${ }^{4-6}$. The IL-1-based genetic test was heavily marketed despite multiple studies that questioned its diagnostic utility ${ }^{3,4}$ and weak and contradictory elements in the supporting literature ${ }^{6}$. MMP8 is a ubiquitous inflammatory molecule, and inflammation contributes to both disease initiation and the healing process. Thus, aMMP8 would have a pivotal role in all phases of inflammation, which would hinder its predictive value $^{3}$. Despite the many potential markers and tests that exist, only a few have verified clinical usefulness, and the Primer provides a balanced overview of those with proven utility or promise.
Denis F. Kinane and Panagiota G. Stathopoulou are at the University of Pennsylvania School of Dental Medicine, 240 South 40th Street, Philadelphia,

Pennsylvania 19104, USA.

Panos N. Papapanou is at Columbia University College of Dental Medicine, New York, New York, USA.

Correspondence to D.F.K. dean@dental.upenn.edu

doi: $10.1038 /$ nrdp. 2017.70

Published online 14 Sep 2017

1. Kinane, D. F., Stathopoulou, P. G. \& Papapanou, P. N. Periodontal diseases. Nat. Rev. Dis. Primers 3, 17038 (2017).

2. Sorsa, T., Gieselmann, D., Arweiler, N. B.

\& Hernández, M. A quantitative point-of-care test for periodontal and dental peri-implant diseases. Nat. Rev. Dis. Primers 3, 17069 (2017).

3. Buduneli, N. \& Kinane, D. F. Host-derived diagnostic markers related to soft tissue destruction and bone degradation in periodontitis. J. Clin. Periodontol. 38 (Suppl. 11), 85-105 (2011).

4. Huynh-Ba, G., Lang, N. P., Tonetti, M. S. \& Salvi, G. E. The association of the composite IL-1 genotype with periodontitis progression and/or treatment outcomes: a systematic review. J. Clin. Periodontol. 34, 305-317 (2007).

5. Hodge, P. J., Riggio, M. P. \& Kinane, D. F. Failure to detect an association with IL1 genotypes in European Caucasians with generalised early onset periodontitis. J. Clin. Periodontol. 28, 430-436 (2001).

6. Diehl, S. R., Kuo, F. \& Hart, T. C. Interleukin 1 genetic tests provide no support for reduction of preventive dental care. J. Am. Dent. Assoc. 146, 164-173 (2015).

Competing interests

The authors declare no competing interests.

How to cite this article

Kinane, D. F., Stathopoulou, P. G. \& Papapanou, P. N. Authors' reply: Predictive diagnostic tests in periodontal diseases. Nat. Rev. Dis. Primers 3, 17070 (2017). 